\title{
Seguridad, eficacia y tolerabilidad de un novedoso apósito de gel de silicona para uso posoperatorio en cirugía dermatológica
}

\author{
Safety, efficacy and tolerability of a novel silicone gel dressing for \\ postoperative use in dermatological surgery
}

Matthias Sandhofer ${ }^{1}$, Patrick Schauer ${ }^{2}$

\begin{abstract}
RESUMEN
Durante muchos años, el gel de silicona ha tenido un rol primario en el tratamiento y la prevención de cicatrices anómalas en forma de cicatrices hipertróficas y queloides luego de la epitelización. Los autores publican los hallazgos preliminares sobre el uso de un novedoso apósito de gel de silicona grado médico que forma una película y que fue aprobado para ser usado en heridas abiertas y en lesiones de la piel como único tratamiento y en combinación con otros tratamientos previos a la reepitelización. Un estudio observacional de 105 pacientes analizó la efectividad del gel de silicona en la estimulación de la epitelización acelerada, la reducción de la respuesta inflamatoria y la prevención de la formación de cicatrices. El estudio se realizo ces. El estudio se realizó en una variedad de intervenciones quirúrgicas dermatológicas. Las observaciones de los autores confirmaron el rol de la silicona en la aceleración de la cicatrización de heridas, la prevención de la formación de cicatrices y la utilidad de este nuevo apósito de silicona que forma una película cuando se lo combina con otras modalidades de tratamiento. (SKINmed. 2012; 10:S1S7)

Palabras claves: gel de silicona, cicatrices hipertróficas, queloides.
\end{abstract}

\begin{abstract}
For many years, silicone gel has played a primary role in the treatment of prevention of abnormal scars in the form of after epithelialization. The authors publish the pre-elimination findings use of a novel medical grade silicone gel dressing that form a film that was approved for use in open wounds and in skin as the only treatment and in combination with other treatments prior to re-epithelialization. An observational study of 105 patients analyzed the effectiveness of silicone gel in the stimulation of accelerated epithelialization, reducing the inflammatory response and preventing the formation of scars.

The study was conducted on a variety of dermatological surgeries. The authors' observations confirmed the role of silicone in acceleration of wound healing, prevention of the formation of the usefulness of this new silicone dressing that forms a film it is combined with other forms of treatment.
\end{abstract}

Keywords: silicone gel, hypertrophic scars, keloids.

REVISTA ARGENTINA DE CIRUGÍA PLÁSTICA 2019;25(2):93-99. HTTPS://DOI.ORG/10.32825/RACP/201902/0093-0099

\section{INTRODUCCIÓN}

Cuando se produce una alteración de la integridad de la piel por una herida o una intervención quirúrgica, las cicatrices resultantes pueden ser estéticamente desagradables o inclusive llegar a la desfiguración, y también pueden estar acompañadas de picazón, dolor y trastornos del sueño. También pueden suscitarse limitaciones funcionales debido a las contracciones de deformación según cuál sea el área afectada y la intensidad de la formación de la cicatriz. Las potenciales consecuencias psicosociales incluyen depresión, limitaciones en la realización de actividades cotidianas, baja autoestima, trastorno de estrés postraumático y estigma-

1. Fundador y Presidente Honorario de la Academia Austríaca de Cirugía Cosmética y Medicina Estética (AACSM). Linz, Austria

2. Médico especialista de la piel. Passau, Alemania

$\triangle$ Correspondencia: Dr. Matthias Sandhofer, Starhembergstrasse 12/3, 4020 Linz, Austria.dr.matthias@sandhofer.at

Los autores no declaran conflictos de intereses

Recibido: 24/08/2019 / Aceptado: 26/08/2019 tización. Todas estas consecuencias de la formación de cicatrices anómalas llevan a una peor calidad de vida ${ }^{1}$. Los médicos que se especializan en intervenciones estéticas emplean una amplia gama de procedimientos quirúrgicos dermatológicos con avances tecnológicos en las técnicas y equipamientos que actualmente permiten obtener un resultado visual y funcionalmente predecible. Consecuentemente, el límite entre los resultados aceptables e inaceptables ha quedado bien definido, tanto de la perspectiva del paciente como de la del médico. Esto significa que el médico no solo debe estar atento durante el procedimiento, sino que también debe estar activamente involucrado en el proceso de cicatrización posoperatorio para asegurar esos resultados predecibles.

El objeto de esta evaluación fue la obtención de datos preliminares sobre la eficacia, seguridad y tolerabilidad de un nuevo apósito de gel de silicona para el tratamiento de heridas y su efecto en la epitelización acelerada y en la prevención de cicatrices anómalas.

\section{USO DE SILICONA EN EL TRATAMIENTO \\ DE CICATRICES Y EN LA PREVENCIÓN \\ DE CICATRICES ANÓMALAS}

A inicios de los 80, la silicona se utilizaba para el tratamiento de cicatrices ${ }^{3}$, especialmente cicatrices hiper- 
TABLA 1. Número y motivo de la intervención ( $n=105)$.

\begin{tabular}{|l|c|}
\hline Motivo de la intervención & $\mathbf{n}$ \\
\hline Eliminación de tatuajes & 22 \\
\hline Tratamiento de cicatrices hipertróficas & 15 \\
\hline Tratamiento de queloides & 2 \\
\hline Tratamiento de cicatrices por acné & 15 \\
\hline Tratamiento de arrugas & 16 \\
\hline Extirpación de nevos & 6 \\
\hline Extirpación de lentigos & 1 \\
\hline Extirpación de melanomas & 4 \\
\hline Extirpación de queratosis & 3 \\
\hline Extirpación de carcinoma basocelular & 1 \\
\hline Estiramiento de la piel (rostro) & 5 \\
\hline Cirugía de párpados & 3 \\
\hline Liposucción & 3 \\
\hline Cirugía venosa & 2 \\
\hline Tratamiento de heridas traumáticas & 2 \\
\hline Estiramiento de la pared abdominal & 2 \\
\hline Tratamiento de verrugas & 1 \\
\hline Mamoplastia & 1 \\
\hline Tratamiento de ojeras & 1 \\
\hline Alopecia cicatrizal & 1 \\
\hline Nevo epidérmico verrugoso inflamatorio lineal & 1 \\
\hline Tratamiento de quemaduras & 1 \\
\hline
\end{tabular}

tróficas y queloides. En virtud de los resultados positivos obtenidos ${ }^{4,5}$, este tratamiento, en forma de láminas de gel de silicona, se transformó rápidamente en el estándar de la cirugía plástica ${ }^{2}$. Su efectividad cuando se la utiliza para la prevención de cicatrices también se confirmó repetidamente ${ }^{6-8}$. Por ello, las Recomendaciones Clínicas Internacionales para el Tratamiento de Cicatrices ${ }^{2}$ aconsejan el uso de láminas de gel de silicona como primera opción para el tratamiento y la prevención de cicatrices hipertróficas y queloides. El desarrollo adicional de la tecnología de silicona y la introducción de productos autosecantes que se expenden con un dosificador posibilitaron superar significativas desventajas y limitaciones asociadas a la aplicación de las láminas. Resultó más fácil aplicar adecuadamente la lámina de silicona, especialmente en áreas de la piel expuestas a movimientos intensivos, y la apariencia cosmética mejoró significativamente, lo cual llevó a una mayor adherencia?. Además de la mejor adherencia por parte de los pacientes, el gel de silicona permite la aplicación de antimicrobianos de uso tópico, protectores solares o maquillaje sobre el gel una vez que se seca. Los estudios clínicos actuales y la experiencia clínica publicada muestran que el gel, que se expende con un dosificador y que se seca con el aire, tiene el mismo efecto que las láminas de gel de silicona tradicionales ${ }^{8,10,11}$ en lo que se refiere al favorecimiento de la cicatrización de heridas durante el tratamiento de cicatrices y en la prevención de la formación de cicatrices anómalas.

\section{CÓMO FUNCIONA ESTE NUEVO APÓSITO} DE GEL DE SILICONA PARA LAS HERIDAS

El gel de silicona se aplica en el área afectada de la piel y forma una lámina protectora flexible que es permeable a los gases, pero es semioclusiva. Cubre la piel lesiona-
TABLA 2. Frecuencia y tipo de intervenciones.

\begin{tabular}{|l|c|}
\hline Tipo de intervención & $\mathbf{n}$ \\
\hline Láser fraccional de CO2 (Lutronic) & 34 \\
\hline Láser Q-Switch & 24 \\
\hline Láser fraccional a 1550 mm (Mosaic) & 23 \\
\hline Escisiones & 17 \\
\hline Inyección de 5-FU/triamcinolona 16 & 16 \\
\hline Radiofrecuencia fraccional (Intracel-FAKIR) & 10 \\
\hline Dermoabrasión & 4 \\
\hline Cauterización & 1 \\
\hline Peeling químico & 1 \\
\hline
\end{tabular}

TABLA 3. Escisiones quirúrgicas en detalle ( $n=17)$.

\begin{tabular}{|l|l|}
\hline Escisiones quirúrgicas & $\mathrm{n}$ \\
\hline Escisión de nevos & 6 \\
\hline Estiramiento de la piel (rostro) & 3 \\
\hline Extirpación de melanoma & 3 \\
\hline Cirugía de párpados & 2 \\
\hline Extirpación de carcinoma basocelular & 1 \\
\hline Mamoplastia & 1 \\
\hline Extirpación de lentigo & 1 \\
\hline
\end{tabular}

da pero no penetra en la epidermis o en la dermis. De acuerdo con los estudios más recientes sobre cicatrices anómalas, las láminas protectoras de silicona restauran la función natural de barrera de la epidermis y reducen la pérdida de agua transepidérmica en el lugar de la herida. Esta pérdida de agua y la resultante deshidratación se consideran factores críticos detrás de la producción excesiva de fibras de colágeno y la consecuente formación de una cicatriz anómala?

Dado que los apósitos semioclusivos sin silicona han demostrado ser menos efectivos en el tratamiento de cicatrices $^{12}$, uno puede suponer que la silicona posee otras propiedades que favorecen la cicatrización de heridas. Los resultados de los estudios clínicos indican que el gel de silicona modula los niveles del factor de crecimiento de fibroblastos $\beta$. La formación de tejido cicatricial hipertrófico que contiene fibroblastos histológicamente normales se evita mediante la modulación de la expresión de los factores de crecimiento. Los datos recabados hasta el momento abonan la hipótesis de que las propiedades de las sustancias de la silicona que favorecen la cicatrización de heridas se basan en la teoría de que corrigen una falta o exceso existente de hormonas del crecimiento que controlan el proceso de reparación de los tejidos ${ }^{13}$. En los procedimientos de ablación o exfoliación, el apósito de gel de silicona genera un entorno de cicatrización protector y húmedo.

\section{EL TIEMPO COMO FACTOR EN EL TRATAMIENTO DE HERIDAS CON SILICONA}

El conocimiento obtenido a partir de los estudios sugiere que el momento en que se trata una herida tiene un efecto significativo en la posible aparición de una cicatriz anómala ${ }^{14-16}$; por lo tanto, el objetivo debería ser alcanzar la epitelización de la herida lo más rápidamente posible para minimizar la formación de la cicatriz. Esto es particularmente cierto en el caso de las heridas provocadas por quemaduras y otras lesiones su- 


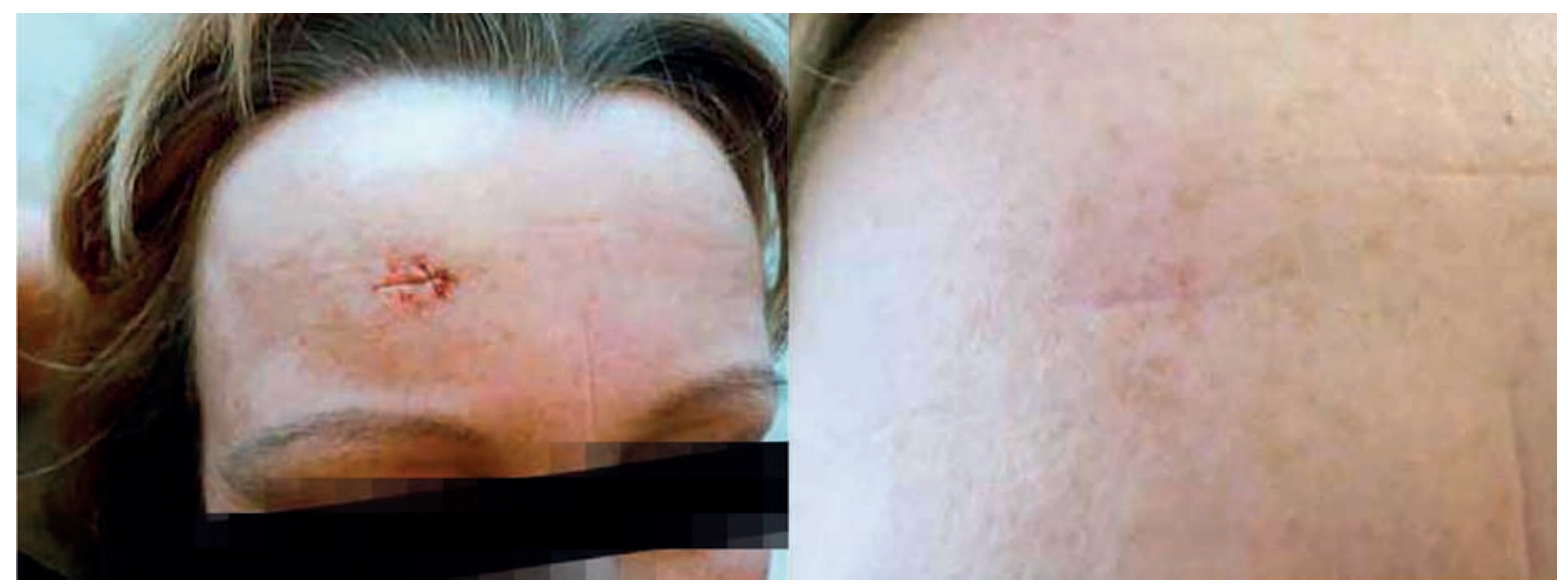

Figura 1. Cicatriz inmediatamente después de la incisión y luego de 8 semanas de tratamiento con Stratamed como único tratamiento. Publicado por primera vez en 2010 en www.stratamed.com.

perficiales de la epidermis, donde la epitelización se demora sin tratamiento adicional; sin embargo, antes de la introducción de este nuevo preparado de silicona, los productos de gel de silicona autosecantes no se indicaban hasta tanto la herida hubiese cicatrizado y la formación epitelial se hubiese completado, y ello producía un retraso del tratamiento de hasta varias semanas. Este nuevo apósito de gel de silicona es potencialmente apropiado para prevenir la formación de cicatrices, ya que también puede usarse inmediatamente en heridas abiertas y en la piel dañada.

Además del tratamiento temprano para la prevención de cicatrices, los procedimientos de ablación y exfoliación dermatológica requieren un entorno protector y húmedo inmediato para facilitar una correcta cicatrización de las heridas. Los efectos físicos de este nuevo gel son potencialmente apropiados para estas indicaciones, ya que también se puede usar inmediatamente después de la intervención.

\section{MÉTODOS}

Los siguientes casos ejemplifican el desempeño de un nuevo producto a base de silicona (Stratamed) en una serie de procedimientos estándar realizados en el Zentrum für Haut, Ästhetik, Laser und Venen en Linz, Austria, y en la Dermatologische Gemeinschaftspraxis, Asthetische Medizine en Passau, Alemania (incluyendo procedimientos ablativos y quirúrgicos). Stratamed es un gel de silicona que se expende mediante un dosificador (Stratpharma AG, Basilea, Suiza) y forma una lámina protectora autosecante. Consiste en polímeros de silicona inertes y puede aplicarse directamente en heridas abiertas y en áreas de la piel donde la integridad esté debilitada. Este estudio describe nuestras observaciones con este producto en una cohorte de 105 pacientes.

\section{SELECCIÓN DE PACIENTES}

De 2009 a 2010, monitoreamos el desempeño de Stratamed luego de varios tratamientos invasivos en 105 pacien- tes en una serie de casos en la clínica de los autores. Las Tablas 1, 2 y 3 describen los diferentes tipos de patologías tratadas y las técnicas aplicadas en una serie de pacientes.

La Tabla 1 muestra el número y los motivos de las intervenciones de los 105 pacientes. Tres de ellos fueron sometidos a más de una intervención. La Tabla 2 describe la frecuencia (130) y los tipos de intervenciones realizadas, con varios pacientes que fueron sometidos a una combinación de técnicas. La Tabla 3 describe específicamente el número y los motivos para la realización de las escisiones en 17 pacientes que no tenían cicatrices antes del procedimiento.

Las variables de los pacientes tales como la edad, sexo y otras variables de confusión, incluyendo el tipo de piel según la escala de Fitzpatrick, no han sido descriptas porque el objetivo de este estudio era observar el desempeño general de este nuevo gel de silicona en muchos tipos de intervenciones quirúrgicas y medir el grado de satisfacción de la paciente con el nuevo producto. Además, no hubo un grupo de referencia para comparar las prácticas posoperatorias habituales con este nuevo tratamiento. En lugar de ello, todos los pacientes fueron sometidos a nuestro protocolo habitual para el tratamiento de heridas con el agregado del nuevo gel.

\section{DESCRIPCIÓN DE LOS PROCEDIMIENTOS}

Para la exfoliación con láser, el gel de silicona se aplicó como coadyuvante de nuestro protocolo habitual para el tratamiento de las heridas posterior al uso de láser. Es decir, a todos los pacientes se les recetó un gel hidratante a base de petróleo.

A algunos pacientes, con base en el criterio médico y los factores de riesgo para el paciente, se les recetó también un ungüento antibiótico con ácido fusídico como ingrediente activo para ser aplicado dos veces al día por los primeros 2 o 3 días; por lo tanto, nuestro tratamiento habitual no se vio modificado salvo por el agregado del gel de silicona como primera capa de contacto.

Luego de las escisiones quirúrgicas y la resutura, el gel de silicona se aplicó por primera vez inmediatamente después del cierre de la herida, y luego se cumplió nues- 


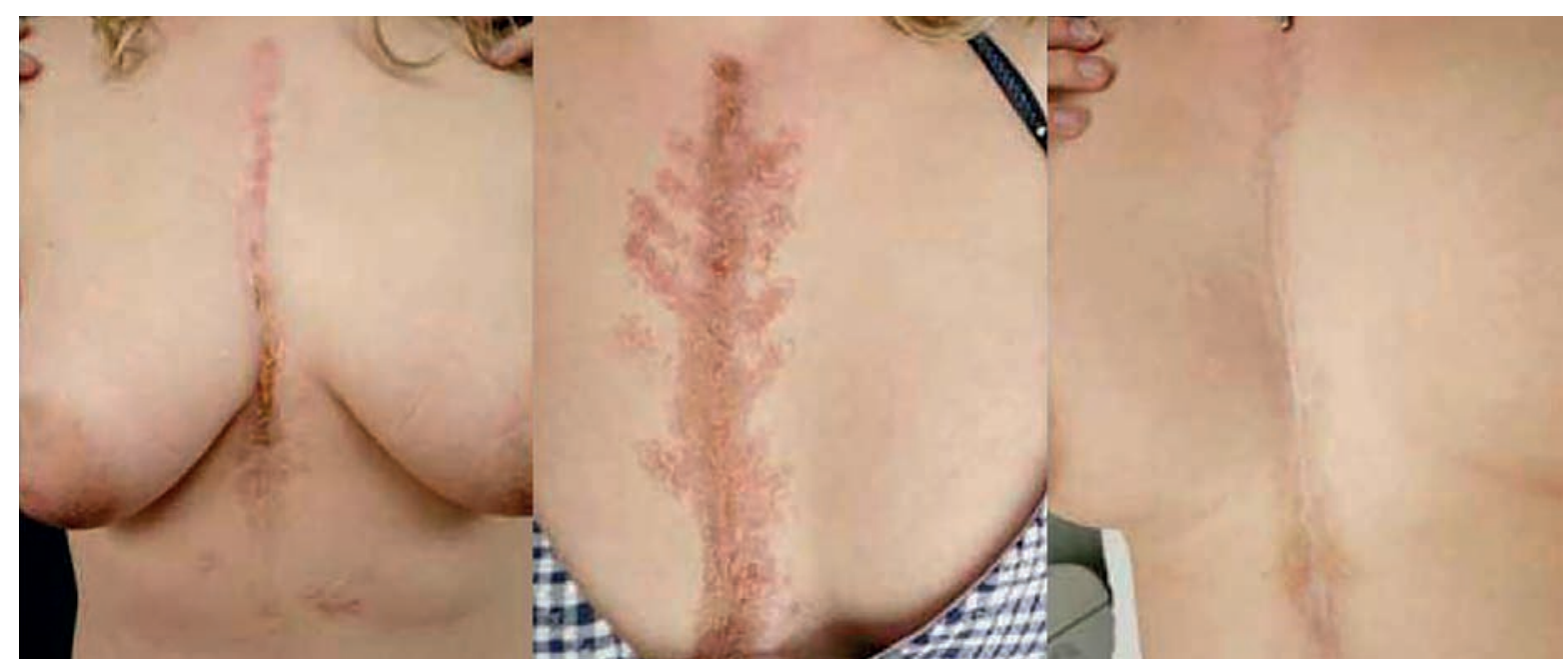

Figura 2. Cicatriz hipertrófica existente luego de una cirugía torácica antes, durante y después de un tratamiento de 10 semanas con Stratamed en combinación con el láser fraccional de $\mathrm{CO}_{2}$ /Mosaic y tratamiento de radiofrecuencia. Publicado por primera vez en 2010 en www.stratamed.com

tro protocolo habitual de tratamiento de heridas, es decir, una combinación de la revisión regular con o sin apósitos para las heridas. En esta serie de casos no se utilizó ningún tratamiento profiláctico adicional para las cicatrices. En cada caso, el exceso de exudado o los fluidos de la herida se secaron manualmente antes de la aplicación del gel de silicona. Según el área de la piel y la herida a ser tratada, el gel de silicona se aplicó con o sin un apósito protector. Los factores que determinaron el uso de un apósito protector no se apartaron de nuestro criteriohabitual para la aplicación de apósitos en el período posoperatorio.

A los 17 pacientes que requirieron tratamiento de heridas anormales existentes -cicatrices hipertróficas y queloides (ambas luego de la aplicación de inyecciones de 5-fluoro-uracil (FU)/triamcinolona así como luego del uso de láseres fraccionales y sistemas de radiofrecuencia fraccional) - se les indicó la aplicación posoperatoria del gel de silicona.

En todos los procedimientos dermatológicos, luego de la primera aplicación en la clínica, los pacientes continuaron utilizando el gel en casa.

La duración promedio del tratamiento para aquellos pacientes que usaron el gel de silicona fue de 3 meses. El seguimiento de estos 105 pacientes duró de 1 a 12 meses, según el procedimiento realizado, pero de conformidad con nuestro seguimiento habitual. Todos los pacientes fueron fotografiados durante el seguimiento (Figuras 1 a 6). Los autores observaron el desempeño general del producto de silicona y evaluaron subjetivamente estos resultados, con base en la experiencia clínica previa y en las expectativas. Además, se registraron las opiniones subjetivas de los pacientes.

\section{RESULTADOS Y OBSERVACIONES}

En los casos de procedimientos de exfoliación, se observaron varios aspectos positivos. Hubo una reducción en

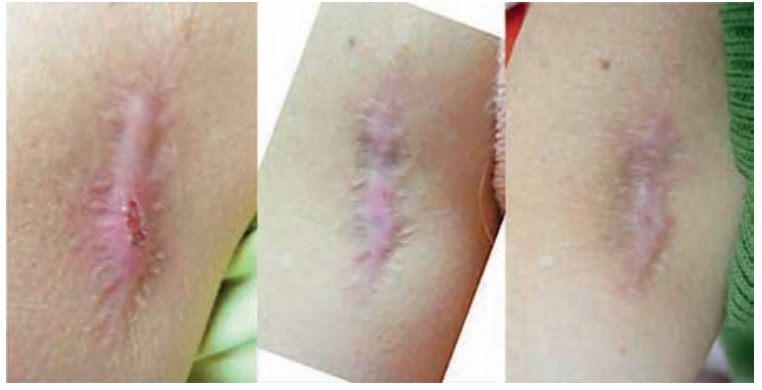

Figura 3. Cicatriz hipertrófica existente luego de la escisión de un melanoma antes del tratamiento y luego de 2 y 7 semanas de tratamiento con Stratamed en combinación con inyecciones de 5-FU/corticosteroide. Publicado por primera vez en 2010 enwww.stratamed.com.

la respuesta inflamatoria, tanto en tiempo como en severidad; se observó una cicatrización precisa de la herida y no se observaron casos de irritación (enrojecimiento). También observamos una cicatrización más rápida o reepitelización de la superficie de la herida con resultados visiblemente satisfactorios que comenzaron luego de 7 días, en comparación con lo que observamos con nuestro tratamiento habitual (Figuras 4, 5 y 6). La lámina protectora formada por el gel de silicona en estos casos de exfoliación no interfirió con la granulación de la herida. Además, luego de la remoción del detrito inicial, no se observó formación de granulación anormal. Se procedió a exfoliar el detrito derivado del tratamiento de $\mathrm{CO}_{2}$ fraccional luego de 3 a 5 días.

Los pacientes refirieron que hubo una significativa reducción del enrojecimiento, la picazón y la sensación de tirantez en estos casos de procedimientos ablativos o semiablativos. El gel desilicona se describió como no disruptivo y resultó estéticamente tolerable para los pacientes, especialmente en los casos de aplicaciones faciales.

Un paciente sin antecedentes de infección de herpespor el uso de laser fraccional de $\mathrm{CO}_{2}$ manifestó que sufrió 


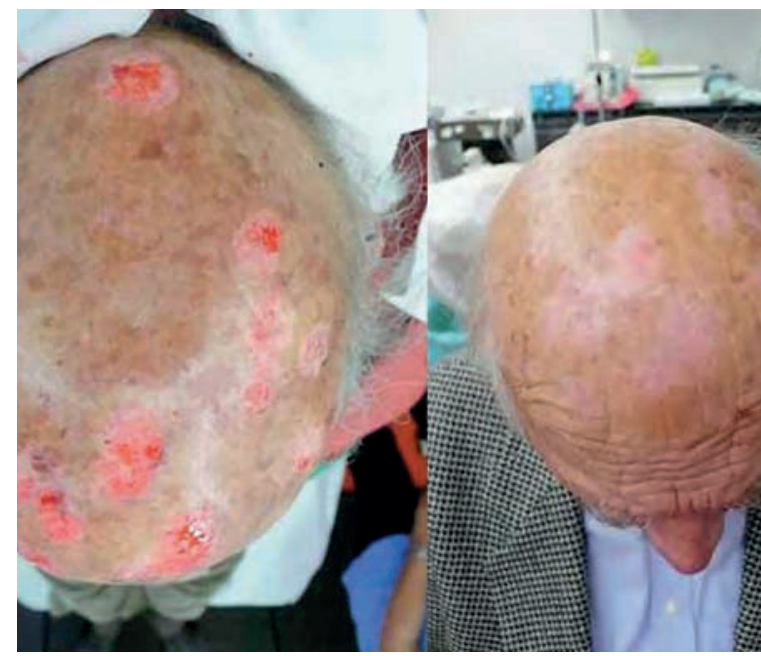

Figura 4. Dermoabrasión durante el tratamiento y luego del tratamiento de 4 semanas con Stratamed como único tratamiento. Publicado por primera vez en 2010 en www.stratamed.com

una erupción herpética en un área bastante amplia, la cual se trató exitosamente con aciclovir sistémico. Del punto de vista de nuestra experiencia, no resulta extraño que surja una erupción herpética luego de un trauma severo en la piel como el derivado del tratamiento con láser fraccional de $\mathrm{CO}_{2}$.

No se observaron otros efectos secundarios derivados del uso del gel de silicona luego de los procedimientos con láser.

En los casos de escisiones quirúrgicas, observamos que la reepitelización y la formación de cicatrices superó nuestras expectativas médicas (Figura 1). La aplicación del gel de silicona representó una mejoría en el proceso de atención posoperatoria de los pacientes, quienes pudieron realizar la aplicación de forma independiente, lo cual significó que ya no fuera necesario que visitaran nuestras clínicas (salvo para los seguimientos obligatorios). La adherencia al tratamiento en estos 17 casos fue del $100 \%$, y esta fue definida como el uso del gel de silicona al menos 6 días a la semana.

Todas las recomendaciones de aplicación posoperatoria se siguieron sin excepción.

En un caso de escisión quirúrgica (tras una escisión de un melanoma juvenil en el área del tórax), observamos que se produjo una cicatriz hipertrófica que se trató exitosamente con la aplicación de inyecciones de 5-FU/triamcinolona en combinación con el gel de silicona.

\section{DISCUSIÓN}

Las observaciones generales de nuestra experiencia clínica indican que este nuevo apósito de gel de silicona para las heridas es apropiado para una amplia gama de indicaciones en cirugía dermatológica, especialmente para el tratamiento de heridas abiertas, procedimientos posexfoliación y el uso oportuno para la prevención de cicatrices anómalas. Este gel también resulta apro-

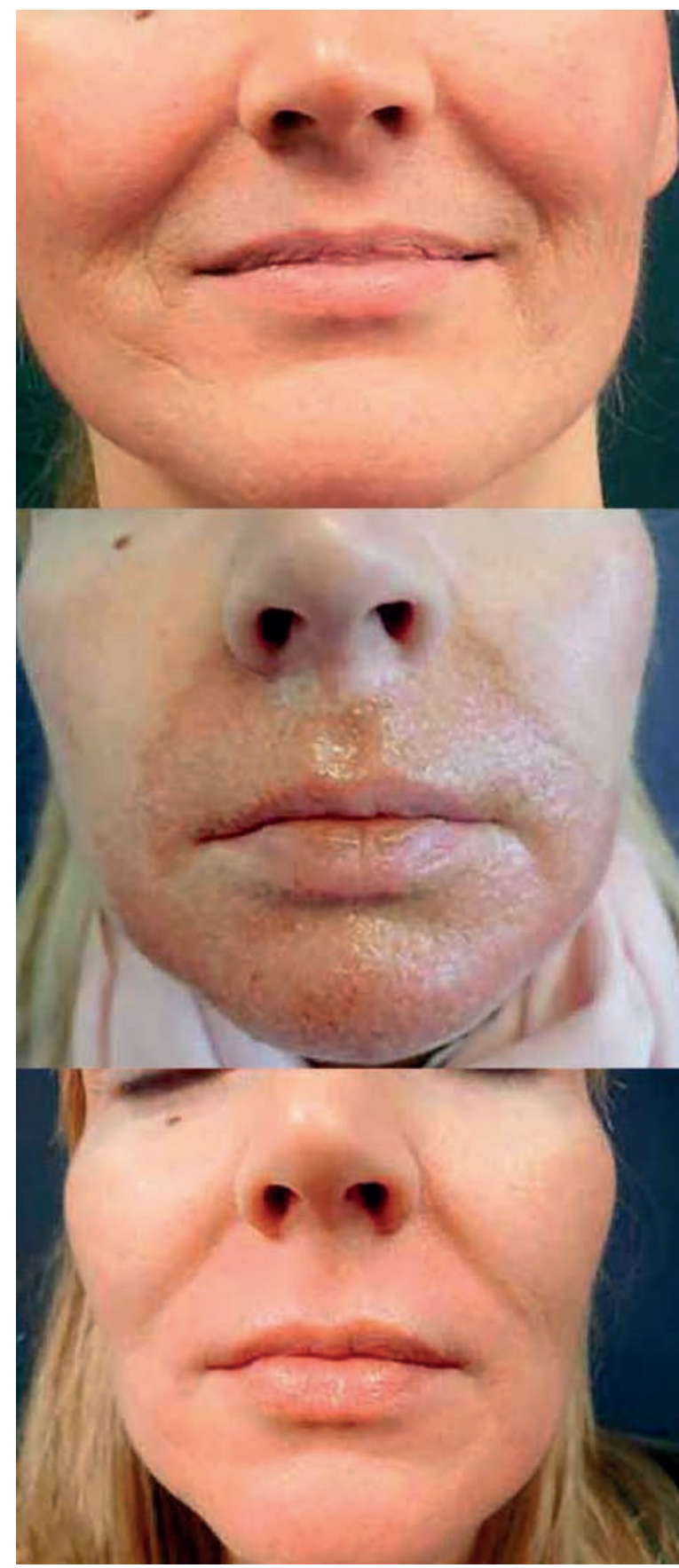

Figura 5. Tratamiento con láser para las arrugas antes, durante (día 0) y después de 10 días de tratamiento con Stratameden combinación conThermage, láser fraccional de $\mathrm{CO}_{2}$ y Botox. Publicado por primera vez en 2010 en www.stratamed.com.

piado como tratamiento de combinación con nuestros protocolos habituales de tratamiento posoperatorio de heridas. El gel de silicona probó ser particularmente útil en posoperatorios de tratamientos con sistemas fraccionales, donde observamos que las heridas erosivas y ablativas quedan mejor protegidas que con los agentes hidratantes a base de petróleo que utilizamos normalmente.

Asimismo, se observó que el proceso de curación estuvo mejor sustentado. Creemos que se debe a que los polímeros de la silicona no tienen un valor de $\mathrm{pH}$ mensu- 


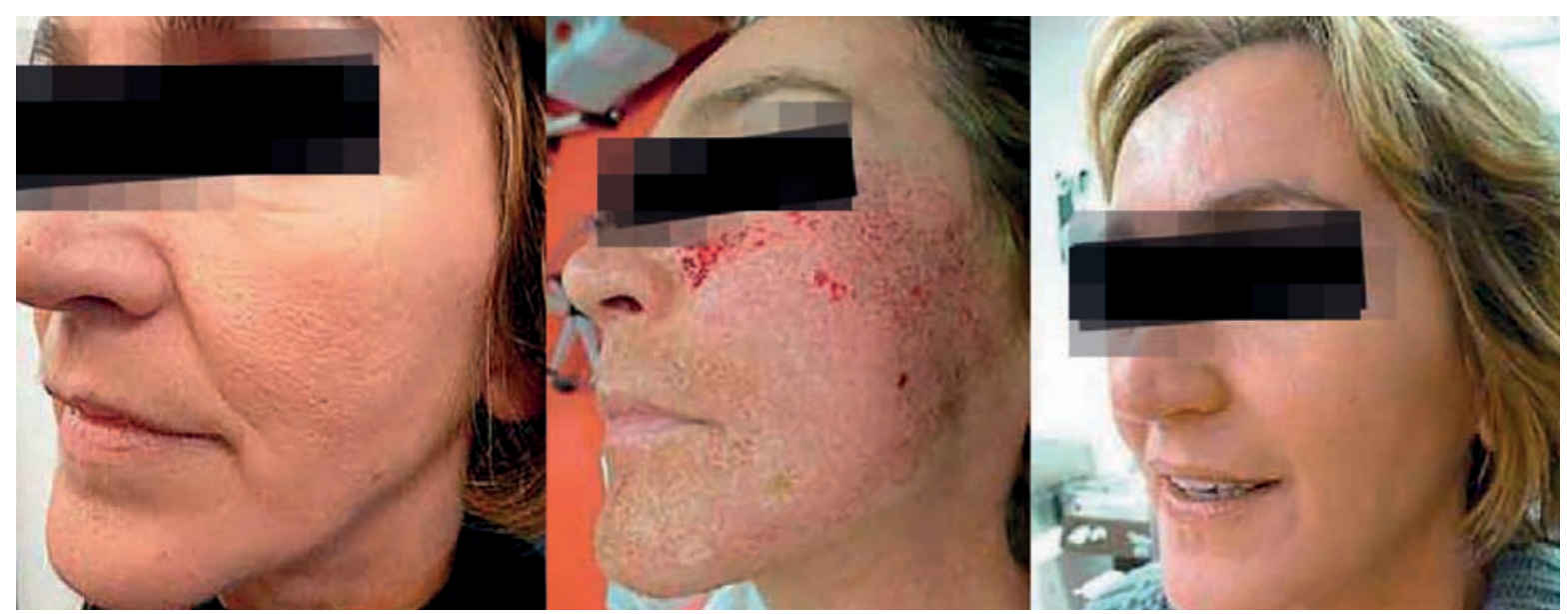

Figura 6. Exfoliaciónde acné con láser antes, durante (día 0) y después de 7 días de tratamiento con Stratameden combinación con Thermage y láser fraccional de $\mathrm{CO}_{2}$. Publicado por primera vez en 2010 enwww.stratamed.com

rable, y por lo tanto esos polímeros no afectan la capa de ácido protector de la piel y no reaccionan con los tejidos epiteliales de reciente formación. El resultado es un entorno favorable para el proceso de cicatrización de la piel que, según observamos en cada caso, lleva a una formación epitelial más rápida.

De acuerdo con la experiencia de nuestro estudio, concluimos que no es necesario adaptar más aún nuestros criterios de tratamiento de heridas existentes cuando se usa gel de silicona, dado que se trata de un tratamiento complementario. La preparación fue bien tolerada, sin excepción, y no se observó sensibilización por contacto ni infecciones relacionadas con el gel. En ningún momento fue necesario retirar la preparación. Dado que el gel es inerte y que a la fecha no se han publicado reacciones adversas al gel de silicona de uso tópico fue posible predecir este resultado. La adherencia de los pacientes fue muy alta y creemos que los factores que contribuyeron a este resultado obedecieron particularmente a la simplicidad de la aplicación, que pudo realizarse en casa sin ningún tipo de dificultad, así como al tipo de clientela tratada en la clínica. Los pacientes atendidos son muy "cosméticamente conscientes", en especial con respecto a la prevención de cicatrices anómalas, y los autores creen que esto lleva a un máximo nivel de adherencia posoperatoria; por lo tanto, la tasa de adherencia observada del $100 \%$ con las escisiones quirúrgicas no causa sorpresa.

\section{LIMITACIONES}

Este estudio observacional tiene importantes limitaciones que deben ser tomadas en cuenta a la hora de interpretar los resultados. El estudio no fue aleatorizado, no tuvo un grupo de referencia, $\mathrm{ni}$ indicaciones habituales para el tratamiento, ni criterios de valoración predefinidos. Ante la ausencia de una metodología experimental rigurosa, no es posible concluir científicamente que nuestras observaciones sean predictivas de los resultados de un estudio comparativo aleatorizado. Con el fin de validar y cuantificar los resultados positivos de estas observaciones iniciales, recomendamos que se realicen estudios clínicos adicionales rigurosos, con herramientas de medición confiables, con pacientes en condición de ciego, evaluadores y un grupo de referencia.

\section{CONCLUSIONES}

Las observaciones preliminares sugieren que este apósito de gel de silicona autosecante de uso tópico para la cicatrización de las heridas es un tratamiento efectivo que contribuye a una más rápida epitelización, a una reducción en la respuesta inflamatoria y a la reducción de la formación de cicatrices anómalas. La facilidad de aplicación y la utilidad de esta nueva forma de apósito de silicona (Stratamed) también simplifica su uso en combinación con otros productos. Puede esperarse una alta adherencia de los pacientes con respecto al uso del gel luego de una amplia gama de intervenciones quirúrgicas dermatológicas.

Declaración: Esta investigación clinica se realizó con el apoyo de Stratpharma AG, Suiza. Los autores no tienen ningún interés económico en Stratpharma AG o Stratamed. 


\section{BIBLIOGRAFÍA}

1. Bayat A, McGrouther DA, Ferguson MW. Skin scarring. BMJ. 2003; 326:88-92.

2. Mustoe TA, Cooter RD, Gold MH, et al. International clinical recommendations on scar management. Plast Reconstr Surg. 2002;110:560-571.

3. Perkins K, Davey RB, Wallis KA. Silicone gel: a new treatment for burn scars and contractures. Burns Incl Therm Inj. 1983;9:201-204.

4. Cruz-Korchin Nl. Effectiveness of silicone sheets in the prevention of hypertrophic breast scars. Ann Plast Surg. 1996;37:345-348.

5. Ahn ST, Monafo WW, Mustoe TA. Topical silicone gel for the prevention and treatment of hyperthrophic scar. Arch Surg. 1991;126:499504.

6. Gold MH, Foster TD, Adair MA, Burlison K, Lewis T. Prevention of hypertrophic scars and keloids by the prophylactic use of topical silicone gel sheets following a surgical procedure in an office setting. Dermatol Surg. 2001;27:641-644.

7. Borgognoni L. Biological effects of silicone gel sheeting. Wound Repair Regen. 2002;10:118-121.

8. Chan KY, Lau CL, Adeeb SM, Somasundaram S, Nasir-Zahari M. A randomized, placebo-controlled, double-blind, prospective clinical trial of silicone gel in prevention of hypertrophic scar development in median sternotomywound. Plast Reconstr Surg. 2005;116:1013-1022.
9. Mustoe TA. Evolution of silicone therapy and mechanism of action in scar management. Aesthetic Plast Surg. 2008;32:82-92.

10. Signorini $M$, Clementoni MT. Clinical evaluation of a new self-drying silicone gel in the treatment of scars: a preliminary report. Aesthetic Plast Surg. 2007;31:183-187.

11. Tobola J, Witkowska-Tobola A, Wlodarkiewicz A. Silicone gel in prevention and treatment of hypertrophic scars and keloids. Dermatologia Estetyczna. 2004;6:323-328.

12. Saulis AS, Chao JD, Telser A, Mogford JE, Mustoe TA. Silicone occlusive treatment of hypertrophic scar in the rabbit model. Aesthet Surg J 2002; 22:147-153.

13. Hanasono MM, Lum J, Carroll LA, Mikulec AA, Koch RJ. The effect of silicone gel on basic fibroblast growth factor levels in fibroblast cell culture. Arch Facial Plastic Surg. 2004;6:88-93.

14. Tandara AA, Mustoe TA. The role of the epidermis in the control of scarring: evidence for mechanism of action for silicone gel. J Plast Reconstr Surg. 2008;61:1219-1225.

15. Upton Z, Lynam E. Treatment innovations in hypertrophic and keloid scars. Wounds International. 2011;2:7-8.

16. Gauglitz GG, Korting HC, Pavicic T, Ruzicka T, Jeschke MG. Hypertrophic scarring and keloids: pathomechanisms, current and emerging treatment strategies. Mol Med. 2011;17:113-125. 